\title{
Prevalence and Antifungal Susceptibility of Candida Species Isolated From Patients Attending Tertiary Care Hospital
}

\author{
${ }^{1}$ Dr Amar C. Sajjan. MD , ${ }^{2}$ Dr. V. V. Mahalakshmi.MD, ${ }^{3}$ Drvinay Hajare, MD \\ 1, 2, 3 Department Of Microbiology,NMC Raichur
}

\begin{abstract}
:
Objectives: Study of prevalence, speciation and antifungal susceptibility of Candida species from samples received by Microbiology Laboratory during the period of one year

Methods: A total of 103 Candida isolates were included in the study. Candida was identified from clinical samples by wet mount, gram stain and culture on SDA. The organisms were further speciated by germ tube test, cornmeal agar morphology, sugar assimilation and fermentation tests and CHROM agar. Antifungal sensitivity was performed by using disc diffusion method.

Results: The prevalence of Candida species was found to be 6.1\%. The most common isolate found to be Candida albicans (65.0\%) followed by non albicans Candida mainly C.tropicalis (24.3\%), and C.krusei (10.7\%). Highest isolates (40.8\%) were from high vaginal swab. The common predisposing factors causing candidiasis were diabeties,pregnancy,prolonged drug intake.. Resistance to commonly used antifungal agents found to be Fluconazole - 12.62\%, Clotrimazole - 7.76\%, Ketoconazole - 0.97\%, Amphotericin-B - 0.97\% and No resistance was seen to Nystatin.

Conclusion: C.albicans is the common cause of candidiasis followed by C.tropicalis and C.krusei. Use of CHROMagar is rapid, technically simple and cost effective compared to time consuming technically demanding expensive conventional method. Antifungal susceptibility testing has to be carried out before starting therapy because of varying susceptibility pattern in different species of Candida.
\end{abstract}

Key words: Antifungal agents; Candida albicans; candidiasis; CHROM agar; .

\section{Introduction}

The genus Candida comprises about 200 species, of which close to 20 has been associated with pathology in human. Candida species are the most common cause of fungal infections worldwide. They can cause a great variety of infections, including simple mucocutaneous to severe invasive infections that can involve virtually any organ. Blood stream infections by Candida are increasingly common, and often are associated with high mortality rates ${ }^{1,2}$. Recently, there has been an increment in the frequency of non-alcicans species of Candida, such as C.glabrata, C.krusei, C.tropicalis, and C.parapsilosis, as causes of fungemia ${ }^{3,4}$.

With the remarkable modern advances in medicine, there has been an increase in the number of immunocompromised individuals who need extensive care in hospitals.

This has resulted in a rise in the incidence of fungal infections, C.albicans is amongst the most common fungal causative agent in superficial and deep seated candidiasis. However, non-albicans Candida species are also being implicated in recent years 5 .

The overall increase in candidemia in recent years is complicated by the emergence of non-albicans Candida (NAC) species as both colonizers and pathogens causing nosocomial fungal blood stream infection (BSI). The NAC species isolated were C.glabrata, C.krusei, C.tropicalis and C.parapsilosis. other species like C.guilliermondii, C.lusitaniae, C.dubliniensis, C.kefyr,C.lipolytica, and C.pelliculosa were occasionally isolated

\section{Material And Methods}

Present study was carried out in the department of Microbiology, Navodaya medical College hospital and Research Centre, Raichur for a period of one year.

Sample size: All the clinical samples (1702) that came to the Department of Microbiology laboratory during the study period constituted the material for the study.

The specimens for laboratory investigation were collected from the clinically suspected cases of candidiasis under strict aseptic precautions. Sterile swabs and bottles were used to collect the appropriate clinical materials. The various clinical specimens collected were oral swabs, ear swabs, vaginal swabs, stool, CSF, sputum, blood, pus, nail scrapings etc. the specimen was subjected to preliminary tests like wet mount, gram stain, culture on SDA and urease test. 
Besides these above specimens for microbiological tests; routine investigations were conducted such as hemogram, urine for albumin and sugar, fasting and post prandial blood sugar, screening the chest, VDRL, and tests for HIV.

Mycological examinations for 103 candida specieswere done: ${ }^{6,11,13 .}$

1) Direct examination:

a. Wet mount

b. Gram's stain

a. Wet Mount:

For direct microscopic examination the specimen was placed on the glass slide then $10 \% \mathrm{KOH}$ (Potassium Hydroxide) was added and coverslip was placed on the specimen. The slide was slightly warmed and gentle pressure was applied over the coverslip to remove the trapped air. Mircroscopic examination was done, first under the low power then under high power.

In case of nail specimen $40 \% \mathrm{KOH}$ is added to a small test tube to which nail clipping was added and incubated overnight for dissolving nail keratin. The specimen was then examined under microscope. ${ }^{11} \mathrm{Attempt}$ was made to identify the pseudohyphae and yeast cells.

b. Gram's stain:Smears were made from the clinincal sample on a clean and grease free glass slide, then heat fixed by just passing the glass slide over the flame.

c. Then thesmear was stained by gram's method and observed under oil immersion objective and looked for the presence of gram-positive oval yeast like budding cells $(2-4 \mu \mathrm{m})$ and / or pseudohyphae.,13

2) Culture: ${ }^{11,14}$ For culture sabouraud's dextrose Agar (SDA) with chloramphenicol was used. The sample was inoculated on SDA slopes and incubated at $25^{\circ} \mathrm{C}$. the slopes were observed regularly daily from 2 days to 3 weeks.

Colonies were identified by the colony characters and by gram's stain. Once the colonies were confirmed speciation was done by following methods.

a. Germ tube

b. Corn meal agar inoculation

c. Sugar fermentation.
d. Sugar Assimilation

e. CHROMagar Candida

Antifungal susceptibility testing:

Antifungal susceptibility testing of the yeast isolates was assessed using the agar diffusion method according to CLSI guidelines.

Disc diffusion method was used in the present study. The antifungal agents used for disc diffusion method are:

1. Amphotericin B (100 IU)

2. Fluconazole $(25 \mu \mathrm{g})$

3. Nystatin $(50 \mu \mathrm{g})$

4. Ketoconazole $(50 \mu \mathrm{g})$

5. Clotrimazole $(10 \mu \mathrm{g})$

To determine whether the isolates tested against amphotericin B,nystatin, fluconazole, ketoconazole, clotrimozole, were susceptible ,intermediate, or resistant; the diameters of zones of inhibition obtained were compared with the standard Zones interpretive break points published by CLSI M44-A2 ${ }^{15}$ guidelines, ROSCO diagnostica ${ }^{16}$

\section{Statistical analysis:}

\section{Results}

Descriptive statistics such as mean, standard deviation (SD), Proportions andgraphs were used to describe the data. Differences between proportions were analyzed using $\chi^{2}$ tests, or by fisher's exact test, if sample sizes were small or unbalanced. A two-tailed p-value $<0.05$ was considered statistically significant.

Table 1: Prevalence of Candida species in clinical specimens

\begin{tabular}{|l|c|c|c|}
\hline \multicolumn{1}{|c|}{ Clinical specimens } & Total No. screened & Total No. of isolates & Percentage \\
\hline HVS & 360 & 42 & 11.7 \\
\hline Sputum & 300 & 21 & 7.0 \\
\hline Oral Swabs & 60 & 12 & 20.0 \\
\hline Pus & 330 & 6 & 1.8 \\
\hline Urine & 550 & 6 & 1.1 \\
\hline Ear Swabs & 15 & 5 & 33.3 \\
\hline Nail & 10 & 5 & 50 \\
\hline Stool & 40 & 5 & 10.0 \\
\hline Conjunctival swabs & 5 & 1 & \\
\hline
\end{tabular}


Prevalence and Antifungal Susceptibility Of Candida Species Isolated From Patients ....

\begin{tabular}{|c|c|c|c|}
\hline Blood & 32 & 1 & 3.1 \\
\hline Total & 1702 & 103 & 6.1 \\
\hline
\end{tabular}

Hundred and three (103) samples out of the 1702 tested positive for candida species giving $(6.1 \%)$

Table 2: Different species of candida isolated

\begin{tabular}{|c|c|c|}
\hline Organism & Total isolates (103) & Percentage \\
\hline C. albicans & 67 & 65.0 \\
\hline C. tropicalis & 25 & 24.3 \\
\hline C. krusei & 11 & 10.7 \\
\hline Total & 103 & 100.0 \\
\hline
\end{tabular}

Candida albicans was the major species accounting for $67(65 \%)$ of the total isolates.non albicans candida constituted 25 (24.3\%) of C. tropicalis, followed by C. krusei 11(10.7\%). All the candida species, isolated from various clinical specimens which were characterized by conventional method, could be identified by CHROM agar.

Table 3: Distribution of isolates among various predisposing factors

\begin{tabular}{|l|c|c|c|}
\hline \multicolumn{1}{|c|}{ Predisposing Factor } & Male & Female & Total (\%) \\
\hline DM & 18 & 16 & $34(33 \%)$ \\
\hline Pregnancy & 0 & 23 & $32(22.3 \%)$ \\
\hline $\begin{array}{l}\text { Prolonged drug intake and secondary to other disease } \\
\text { PD and SD) }\end{array}$ & 12 & 11 & $23(22.3 \%)$ \\
\hline HIV & 4 & 2 & $6(5.8 \%)$ \\
\hline Prolonged contact with & 3 & 2 & $5(4.9 \%)$ \\
\hline Others Total & 4 & 8 & $12(11.7 \%)$ \\
\hline \multicolumn{1}{|c|}{$\chi^{2}=0.772, \mathrm{p}=0.3$, Not Significant } & $\mathbf{1 0 3 ( 1 0 0 \% )}$ \\
\hline
\end{tabular}

In the study, diabetes mellitus was the major predisposing factor constituting 34(33\%) followed by pregnacy $23(22.3 \%)$, prolonged contact with water $5(4.9 \%)$. Other $12(11.7 \%)$ in predisposing factors include, catheterization, stay in ICU, parenteral nurtition, prematurity and low socio-economic status, poor personal hygiene. Even though there is female predominance as compared to male, it is statistically not significant $(\mathrm{p}>0.05)$

Table-4: Distribution of different species of candida among various clinical specimens

\begin{tabular}{|c|c|c|c|c|}
\hline Clinical specimens & C.albicans (\%) & C.tropicalis (\%) & C.krusei (\%) & Total $(\%)$ \\
\hline HVS & $34(81)$ & $6(14.2)$ & $2(4.8)$ & $42(40.8)$ \\
\hline Sputum & $12(57.1)$ & $6(28.6)$ & $3(14.3)$ & $21(20.4)$ \\
\hline Oral Swabs & $5(41.7)$ & $5(41.6)$ & $2(16.7)$ & $12(11.7)$ \\
\hline Pus & $4(66.6)$ & $1(16.7)$ & $1(16.7)$ & $6(5.8)$ \\
\hline Urine & $2(33.3)$ & $3(50)$ & $1(16.7)$ & $6(5.8)$ \\
\hline Ear Swabs & $4(80)$ & 0 & $1(20)$ & $5(4.9)$ \\
\hline Nail & $3(60)$ & $2(40)$ & 0 & $5(4.9)$ \\
\hline Stool & $2(50)$ & $1(25)$ & $1(25)$ & $4(3.9)$ \\
\hline Conjunctival swabs & 0 & $1(100)$ & 0 & $1(1)$ \\
\hline Blood & $1(100)$ & 0 & 0 & $1(1)$ \\
\hline Total & 67 & 25 & 11 & 103 \\
\hline
\end{tabular}

$\mathrm{X}^{2}=8.697, \mathrm{p}=0.013$, significant

The highest number of isolates was from high vaginal swab isolated from vulvovaginitis constituting 42(40.8\%). The other major samples were sputum $21(20.4 \%)$, oral swabs $12(11.7 \%)$, urine $6(5.8 \%)$, pus $6(5.8 \%)$, nail scrapings $5(4.9 \%)$, ear swabs $5(4.9 \%)$, followed by stool $4(3.9 \%)$ and blood $1(1.67 \%)$. 
Figure -1: Distribution of different species of candida among various clinical specimens

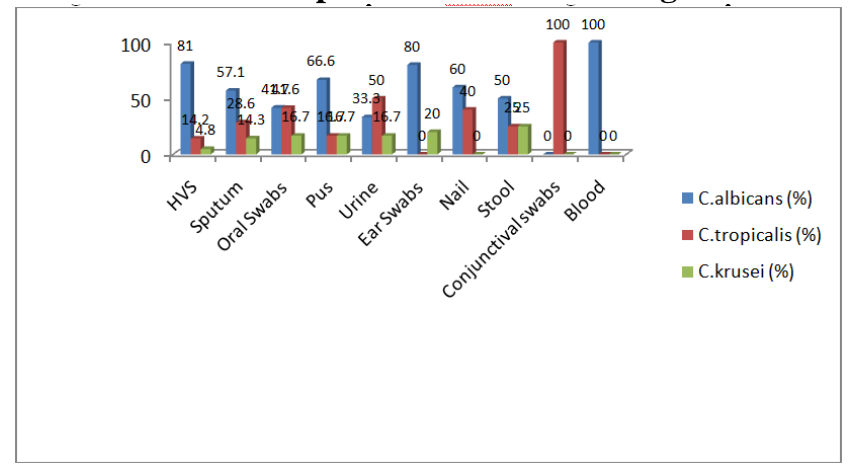

Among 6 urine samples, 2(33.3\%) were C.albicans, 3(50\%) were C.tropicalis and 1(16.7\%) was Candida krusei.

Among 5 nail scrapings, 3(60\%) were C.albicans and 2(40\%) were C.tropicalis.

Among 5 ears swabs, 4(80\%) were C.albicans, 1(18.18\%) one was C.krusei (20\%)

Among 4 samples of stool, 2(50\%) species were C.albicans and one (25\%) each of C.tropicalis and C.krusei.

Table-6: Antifungal Susceptibility Pattern.

\begin{tabular}{|c|c|c|c|c|c|c|c|c|c|c|c|c|c|c|c|}
\hline \multirow{2}{*}{ Species } & \multicolumn{3}{|c|}{ Amphotericin B } & \multicolumn{3}{|c|}{ Nystatin } & \multicolumn{3}{|c|}{ Fluconazole } & \multicolumn{3}{|c|}{ Ketoconazole } & \multicolumn{3}{|c|}{ Clotrimazole } \\
\hline & $\mathbf{S}$ & I & $\mathbf{R}$ & $\mathbf{S}$ & I & $\mathbf{R}$ & $\mathbf{S}$ & I & $\mathbf{R}$ & $\mathbf{S}$ & I & $\mathbf{R}$ & $\mathbf{S}$ & I & $\mathbf{R}$ \\
\hline C. albicans & 66 & $\mathbf{0}$ & 1 & 66 & 1 & $\mathbf{0}$ & 62 & 4 & $\mathbf{1}$ & 66 & $\mathbf{0}$ & 1 & 62 & $\mathbf{0}$ & 5 \\
\hline C. tropicalis & 25 & $\mathbf{0}$ & $\mathbf{0}$ & 24 & 1 & $\mathbf{0}$ & 24 & $\mathbf{0}$ & 1 & 25 & $\mathbf{0}$ & $\mathbf{0}$ & 23 & $\mathbf{0}$ & 2 \\
\hline C. krusei & 11 & $\mathbf{0}$ & $\mathbf{0}$ & 9 & 2 & $\mathbf{0}$ & $\mathbf{0}$ & $\mathbf{0}$ & 11 & 11 & $\mathbf{0}$ & $\mathbf{0}$ & 10 & $\mathbf{0}$ & 1 \\
\hline Total & $\begin{array}{c}10 \\
2\end{array}$ & $\mathbf{0}$ & 1 & 99 & 4 & $\mathbf{0}$ & 86 & 4 & 13 & $\begin{array}{c}10 \\
2\end{array}$ & $\mathbf{0}$ & 1 & 95 & $\mathbf{0}$ & 8 \\
\hline$\chi^{2}$ Value & \multicolumn{3}{|c|}{0.526} & \multicolumn{3}{|c|}{3.13} & \multicolumn{3}{|c|}{11.37} & \multicolumn{3}{|c|}{0.526} & \multicolumn{3}{|c|}{0.02} \\
\hline p-value & \multicolumn{3}{|c|}{0.99} & \multicolumn{3}{|c|}{0.056} & \multicolumn{3}{|c|}{0.001} & \multicolumn{3}{|c|}{0.99} & \multicolumn{3}{|c|}{0.87} \\
\hline
\end{tabular}

In the presemt study, among 103 Candida isolates tested, Ketoconazole and Amphotericin B showed resistance in $0.97 \%$ cases.

For Nystatin, no strains showed resistance and $3.88 \%$ straoms showed intermediate sensitive.

For clotrimazole, $92.23 \%$ strains showed sensitive, $7.76 \%$ strains were resistant and among them $4.85 \%$ were C. albicans, $1.97 \%$ for C. tropicalis, and $0.97 \%$ for C. krusei.

For fluconazole, $83.49 \%$ strains showed sensitive, $12.6 \%$ showed resistance and $3.88 \%$ showed intermediate sensitivity (or dose dependent susceptibility) $(\mathrm{p}=0.001)$

Amphotericin B and Ketoconazole are more sensitive followed by Nystatin, Clotrimazole and Fluconazole. Resistance is seen significantly among non-albicans Candida for Fluconazole $(\mathrm{p}=0.001)$ and Nystatin showed intermediate sensitive $(p=0.056)$.

\section{Discussion:}

The study identified the specific species of Candida that causes infections among patients who attended a tertiary care hospital in North-interior Karnataka, India. The study also determined the in vitro susceptibility of Candida species isolated from the various clinical samples. The antifungal agents tested include Amphotericin B, Nystatin, Fluconazole, Ketoconazole and Clotrimazole.

Candida albicans is by far the most common species causing infection in humans. Candida albicans and non-albicans species are closely related but differ from each other with respect to epidemiology, virulence characteristic and antifungal susceptibility. Distinction between species facilitates the understanding of epidemiology of Candida species particularly regarding the reservoir and mode of transmission which is requirement for the development of effective measures to prevent and control transmission of resistant pathogens.

In the present study it was found that candidiasis can occur at all ages and in both sexes. The youngest in our study was one month old baby while the oldest was 79 years and mean age was 37.3 years.

According to study conducted by Dalal et $\mathrm{al}^{17}$, Akorth et a1, ${ }^{7}$ Puri KJ et al ${ }^{18}$, Jayalaxmi et $\mathrm{al}^{19}$, maximum cases were in the age group of 21-40 years. 
In the present study, females are more commonly affected than males with an incidence of $62(60.2 \%)$ and 4 1(39.8\%) respectively in a ratio of $0.66: 1(\mathrm{M}: \mathrm{F})$. In a similar study by Kandhari $\mathrm{KC}$ et al ${ }^{20}$ at AIIMS, New Delhi, the incidence in females was about $61.2 \%$ while in males it was only $38.8 \%$ with a ratio of $1: 1.57(\mathrm{M}: \mathrm{F})$ and Rizvi MW et al'5also reported female preponderance in their study group with a ratio of $0.85: 1(\mathrm{M}: \mathrm{F})$.

In the present study, Candida species isolated from various clinical samples and the highest was from high vaginal swab $(40.8 \%)$, followed by sputum $(20.4 \%)$, oral thrush $(11.7 \%)$, urine $(5.8 \%)$, pus $(5.8 \%)$, nail scrapings $(4.9 \%)$, ear swabs (4.9\%), stool (3.9\%), blood (1\%) and conjunctival swab (1\%).

In this study Candida isolated from $20.4 \%$ sputum sample which correlates with the study done by yang et $\mathrm{al}^{23}$, who isolated Candida from $16 \%$ of sputum sample)

Dastjdar et $\mathrm{al}^{22}$, in their study found oral thrush in $12 \%$ of patients while in our study $11.6 \%$ which is similarTO the

The predominant species isolated in our study was Candida albicans constituting 65\%. The nonalbicans Candida isolated are Candida tropicalis (24.3\%) followed byCandida krusei $(10.7 \%)$.

Yang et ${ }^{\text {a231 }}$ in Taiwan reported C.albicans $(69.1 \%)$ as the most.common species, followed by C.tropicalis $(12.9 \%)$ and C.krusei was isolated from $0.6 \%$ cases. In a study by Basu et a! ${ }^{21}$ in Delhi, the C.albicans (45.8\%) was the predominant isolate followed by C.tropicalis (24.7\%), C.krusei (7\%). This study is similar to above studies.

Vijaya D etal ${ }^{25}$ isolated 102 clinical isolates of Candida specien from various clinical specimens. The candida species isolated include candida albicans (45.9\%), Cptropicalis (35.29\%) and C.krusei (10.78\%). In this study C.albicans isolated in $65 \%$ cases, a proportion similar to that found by Yong et $\mathrm{al}^{23}$.

In the present study, the results of CHROM agar were exactly paralleled to that of conventional method.

In this study, diabetes mellitus was the most important predisposing factor, accounting for $33 \%$ of the total cases, followed by pregnancy $(22.3 \%)$, prolonged drug intake and secondary to other disease infections which accounted for $22.3 \%$ of cases, HIV (5.8\%), prolonged contact with water (4.9\%) and other predisposing factors constitutes for $11.7 \%$ cases.

In our study, candida species were isolated from patients on prolonged corticosteroids, broad-spectrum antibiotic use and secondary to other diseases accounted for $22.3 \%$ of cases. Among the candida species isolated, $69.7 \%$ were $\mathrm{C}$. albicans, $17 \%$ were C.krusei, and 13 were C.tropicalis.

Arora $\mathrm{D}$ et $\mathrm{al}^{2}$ reported that, $35 \%$ of candidiasiswasdue to prologed use of antibiotics. Pitter $\mathrm{D}$ et $\mathrm{al}^{26}$, in his study, showed that 19 of 29 patients $(66 \%)$ developed candida infection, who received more than two antibiotics before candida infection or some time during their stay within the critical care units.

In present study 6 cases are attributed to HIV Infection, C.tropicalis was isolated from 4 patients and C.albicans isolatedfrom 2 cases.

In a study done by capoor et al $^{24}$ isolated candida apecies from various clinical samples from 6 AIDS patients, them $3(50 \%)$ were C.albicans and 3(50\%) were C.tropicalis. but Baradkar VP et al ${ }^{12}$ found C.albicans $(70 \%)$ as predominant species followed by C.tropicalis in HIV patients with oral candidiasis.

In the present study, candida isolated from $4.8 \%$ cases of onychomycosis.

Sehgal et $\mathrm{al}^{28}$ had observed in their study that nail plate can be involved secondarily in candidiasis and theincidence to be more common in housewives and in professions such as fruit sorters, canners, bartenders and dishwasheres.

Other conprised of $11.7 \%$ in predisposing factors which include, catheterization, stay in ICU, parenteral nutrition, prematurity, poor socioeconomic status, poor personal hygiene and oral contraceptive.

\section{Antifungal susceptibility testing:}

Monitoring antifungal resistance among Candida is useful because apart from tracking and detection of resistance, it also gives clues to emerging threats of new resistant strains. This help in assessing empirical treatment recommendation.

In present study, among 103 candida isolates tested, Ketoconazole and Amphotericin B showed resistance in $0.97 \%$ cases. For Nystatin, no strains showed resistance and $3.88 \%$ strains showed intermediate sensitive, $12.6 \%$ showed resistance and $3.88 \%$ strains showed intermediate sensitive. For clotrimazole, $92.23 \%$ strains showed sensitive, $7.76 \%$ strains were resistant and among them $4.85 \%$ were C.albicans, $1.97 \%$ for C.tropicalis, and $0.97 \%$ for C.krusei. for fluconazole, $83.49 \%$ strains showed sensitive, $12.6 \%$ shoewed resistance and $3.88 \%$ showed intermediate sensitivity (or dose dependent susceptibility) $(\mathrm{p}=0.001$ )

Sobel etal ${ }^{29}$ reported no fluconazole resistance among the 401 Candida albicans recovered from candidal vaginitis in the USA reported fluconazole resistance to be $3.6 \%$ among Candida albicans. ${ }^{30}$

Finding from this study indicate that all theisclates of Candida krusei were resistant to fluconazole. It is well established that Candida krusei is intrinsically resistant to fluconazole. This was also confirmed by Hamza et al ${ }^{31}$ who reported in Tanzania that all isolate of Candida krusei testedwere resistance to flucnazole. 
Vijaya et $\mathrm{al}^{25}$ reported $100 \%$ sensitive to Amphotericin B, Clotrimazole, Nystatin and Ketoconazole and $25 \%$ of C.krusei, $28 \%$ C.tropicalis showed resistance to fluconazole.

To conclude, vulvovaginal candidiasis was the most common clinical type of candidiasis. Commonest age group involved was 20- 49 years and females are more affected than males.

Candida albicans was the most frequently encountered species in the present study, there is rise in the nonCandida albicans isolates from various clinical conditions. the results of CHROM agar were exactly paralleled to that of conventional method. Even though most of the yeasts tested were susceptible to the antifungal agents, periodic antifungal susceptibility testing should be carried out according to CLSI guidelines.

\section{References:}

[1]. Segal E, Elad D. Candidiasis. In: Merz WG, Hay RJ, Topley Wilson's — Microbiology and microbial infection. 10th ed. Medical mycology: London: Hodder Arnold;2007:579-613.

[2]. Arora D, Anand N, Goya G, Kumar R, Gupta P, Santa. Prevalence and risk factors of Candida in cases of candidemia in a tertiary care hospital. International journal of pharmacy and pharmaceutical sciences 2011;3(1): 157-159.

[3]. Wise GJ, Silver DA. Fungal Infections of the Genitourinary System. J Urol 1993;149:1377-88.

[4]. Girmenia C, Martiwo P. Fluconazole and the Changing Epidemiology of candidemia. Clinica.

[5]. Rizvi MW, Malik A, Shahid M, Singhal S. Candida albicans infections in a north Indian tertiary care hospital: Antifungal resistance pattern and role of SDS-PAGE for characterization. Biology and Medicine 2011;3(2) Special Issue: 176-181.

[6]. Chakrabarti A, Shivaprakash MR. Microbiology of systemic fungal infections. J Postgrad Med 2005;51;16-20.-

[7]. Akortha EE, Nwaugo VO, Chikwe NO. Antifungal resistance among Candida species from patients with genitourinary tract isolated Benin City, Edo state, Nigeria. African Journal of Microbiology Research 2009;3(1 1).

[8]. Koneman FW, Allen SD, Janda WM, Wine WC, Schreckenberger PC, Procop GW et al. Koneman's Color Atlas and Textbook of Diagnostic Microbiology. 6th ed. Philadelphia: Lippincott Williams and Wilkins; 2006.

[9]. Chakrabarti A, MR Shivprakash, Venugopal PV, Venugopal TV. National

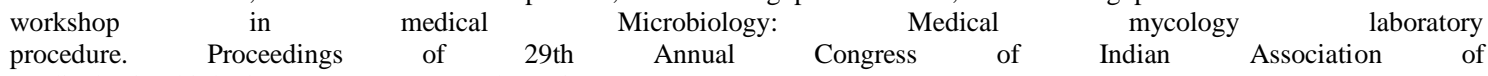
$\begin{array}{llll}\text { Medical Microbiologist; } 2005 \text { Oct 19-20; Chennai. } & & & \\ \text { Hageage GJ, Harrington } & \text { BJ. Use of Calcoflor white in clinical Mycology. }\end{array}$

Lab Med 1984; 15:109-12.
[11]. Milne LIR. Fungi. In: Colle JG, Frase AG, $\quad$ Marmion BP,

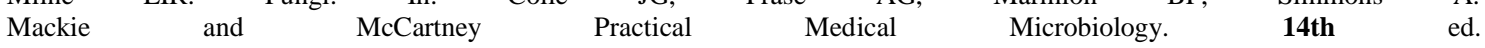
Edinburgh: Churchill Livingstone; 2007:695-717.
Baradkar VP, Mathur M, Kumar S. Hichrom Candida agar for identification of Candida species. Indian J Clin Microbiol 1996;

[12]. Baradkar VP, Mathur M, Kumar S. Hichrom Candida agar for identification of Candida species. Indian J Clin Microbiol 1996;
[13]. Chander J. Textbook of Medical Mycology. Ed.3.

[13]. Chander J. Textbook of Medical Mycology. Ed.3. New Delhi: Mehta;

[14]. Forbes BA, Sahm DF, Weissfeld AS. Laboratory methods in basic Mycology. In: Bailey and Scott's Diagnostic Microbiology. 12th Ed. St. Louis: Mosby; 2007:629-7.

[15]. Clinical Laboratory Standards Institute (CLSI). Method for antifungal disk diffusion Susceptibility testing of Yeasts; Approved Guideline- second edition. CLSI document M44-A2 (ISBN 1-56238-703-0). Clinical Laboratory Standard Institute, Waync: Pennsylvania; 2009.

[16]. Susceptibility testing of yeasts [internet]. 2011 [cited 2011 MAR 16]. Available from: http://rosco.dk/gfx!yea.

[17]. Dalal JP, Kelkar SS. Clinical patterns of Candida infections in Bombay. md J Der Ven Lep 1980;46(1):31-32.

[18]. Puri KJ, Madan A, Bajaj K. Incidence of various causes of vaginal discharge among sexually active females in age group 20-40 years. md J Der Ven Lep 2003;69(2):122-125.

[19]. Jayalaxmi J, Ganapathy M, Nirmala Devi TN. Vulvovaginal candidiasis among women: a surgery. Antiseptic 2004;101(1):16-18

[20]. Khandari KC, Rama KM Rao. Clinical and laboratory studies on cutaneous candidiasis. md J Der Ven Lep 1969;35(2):102-107.

[21]. Basu S, Gugnani HC, Joshi S, Gupta N. Distribution of Candida species in different clinical sources in Delhi, India, and proteinase and phospholipase activity of Candida albicans isolates. Rev Iberoam Micol 2003;20:137- 140.

[22]. Dastidar SG, Desai SC, Purendare MD. Candidiasis in Bombay. J Post Med 1966;12:187.

[23]. Yang YL, Hsieh LY, Wang AH, Lo Hi. Characterization of Candida species from different population in Taiwan. Mycopathologia 2011; 172(2):131-9.

[24]. Capoor MR, Nair D, Deb M, Verma PK, Srivastava L, Aggarwal P. Emergence of non-albicans Candida species and antiftingal resistance in tertiary care hospital. J Infect Dis 2005;58:344-348.

[25]. Vijaya D, Harsha TR, Nagaratnamma T. Candida speciation using chrom agar. Journal of Clinical and Diagnostic Research 2011 ;5(4):755-757. .

[26]. Pittet D, Monod M, Suter PM, Frenk E, Auckenthaler R. Candida colonization and subsequent infections in critically ill surgical patients. Ann Surg 1994;220(6):751-758.

[27]. Baradkar VP, Karyakarte RP. Isolation and characterization of Candida species in acquired Immunodeficiency syndrome. Indian J Med Microbiol 1999; 1 7(1):42-44.

[28]. Sehgal VN, Jam S. Onychomycosis: clinical perspective. Tnt J Dermtol 2000;39:241 -249.

[29]. Sobel JD, Wiesenfeld HC, Martens M, Danna P, Hooton TM, Rompalo, A. Maintenance fluocnazole therapy for recurrent vulvovaginal candidiasis. N Engi J Med 2004;351:876-883.

[30]. Sobel JD, Zervos M, Reed BD, Hooton TM, Soper D, Nyirjesy P. Fluconazole susceptibility of vaginal isolates obtained from women with complicated Candida vaginitis: clinical implications. Antimicrob Agents Chemother $2003 ; 47: 34-8$.

[31]. Hamza OJ, Matee MIN, Moshi MT, Simon ENM, Mugusi F, Mikx FHM, et al. Species distribution and in vitro antifungal susceptibility of oral yeast isolates from Tanzanian HIV-infected patients with primary and recurrent oropharyngeal candidiasis. BMC Microbiol $2008 ; 8: 135$. 